Article

\title{
Asymmetry and Leverage in Conditional Volatility Models
}

\section{Michael McAleer 1,2,3,4}

1 Department of Quantitative Finance, National Tsing Hua University, Hsinchu 101, Taiwan; E-Mail: michael.mcaleer@ gmail.com; Tel.: +886-3-5162534; Fax: +886-3-5621823

2 Econometric Institute, Erasmus School of Economics, Erasmus University Rotterdam, Burgemeester Oudlaan 50, 3062 PA Rotterdam, The Netherlands

3 Tinbergen Institute, Rotterdam 3000 DR, The Netherlands

4 Department of Quantitative Economics, Complutense University of Madrid, Madrid, 28040, Spain

Received: 18 September 2014; in revised form: 19 September 2014 / Accepted: 19 September 2014 / Published: 24 September 2014

\begin{abstract}
The three most popular univariate conditional volatility models are the generalized autoregressive conditional heteroskedasticity (GARCH) model of Engle (1982) and Bollerslev (1986), the GJR (or threshold GARCH) model of Glosten, Jagannathan and Runkle (1992), and the exponential GARCH (or EGARCH) model of Nelson (1990, 1991). The underlying stochastic specification to obtain GARCH was demonstrated by Tsay (1987), and that of EGARCH was shown recently in McAleer and Hafner (2014). These models are important in estimating and forecasting volatility, as well as in capturing asymmetry, which is the different effects on conditional volatility of positive and negative effects of equal magnitude, and purportedly in capturing leverage, which is the negative correlation between returns shocks and subsequent shocks to volatility. As there seems to be some confusion in the literature between asymmetry and leverage, as well as which asymmetric models are purported to be able to capture leverage, the purpose of the paper is three-fold, namely, (1) to derive the GJR model from a random coefficient autoregressive process, with appropriate regularity conditions; (2) to show that leverage is not possible in the GJR and EGARCH models; and (3) to present the interpretation of the parameters of the three popular univariate conditional volatility models in a unified manner.
\end{abstract}

Keywords: conditional volatility models; random coefficient autoregressive processes; random coefficient complex nonlinear moving average process; asymmetry; leverage

JEL classifications: C22; C52; C58; G32 


\section{Introduction}

The three most popular univariate conditional volatility models are the generalized autoregressive conditional heteroskedasticity (GARCH) model of Engle (1982) [1] and Bollerslev (1986) [2], the GJR (or threshold GARCH) model of Glosten, Jagannathan and Runkle (1992) [3], and the exponential GARCH (or EGARCH) model of Nelson $(1990,1991)$ [4,5]. The underlying stochastic specification to obtain GARCH was demonstrated by Tsay (1987) [6], and that of EGARCH was shown recently in McAleer and Hafner (2014) [7].

These models are important in estimating and forecasting volatility and in capturing asymmetry, which is the different effects on conditional volatility of positive and negative effects of equal magnitude; furthermore, they are purportedly important in capturing leverage, which is the negative correlation between returns shocks and subsequent shocks to volatility. The purpose of the paper is three-fold, namely, (1) to derive the GJR model from a random coefficient autoregressive process, with appropriate regularity conditions; (2) to show that leverage is not possible in the GJR and EGARCH models; and (3) to present the interpretation of the parameters of the three popular univariate conditional volatility models in a unified manner.

The derivation of three well known conditional volatility models, namely GARCH, GJR and EGARCH, from their respective underlying stochastic processes raises two important issues: (1) the regularity conditions for each conditional volatility model can be derived in a straightforward manner; and (2) the GJR and EGARCH models can be shown to capture asymmetry, but they can also be shown to be unable to capture leverage.

The paper is organized as follows. In Section 2, the GARCH, GJR and EGARCH models are derived from different stochastic processes, the first two from random coefficient autoregressive processes and the third from a random coefficient complex nonlinear moving average process. It is shown that asymmetry is possible for GJR and EGARCH, but that leverage is not possible. Issues related to estimation are also discussed, with a view to guaranteeing positive estimates of conditional volatility. Some concluding comments are given in Section 3.

\section{Stochastic Processes for Conditional Volatility Models}

\subsection{Random Coefficient Autoregressive Process and GARCH}

Consider the conditional mean of financial returns as in the following:

$$
y_{t}=E\left(y_{t} \mid I_{t-1}\right)+\varepsilon_{t}
$$

where the returns, $y_{t}=\Delta \log P_{t}$ represents the log-difference in stock prices $\left(P_{t}\right), I_{t-1}$ is the information set at time $t-1$, and $\varepsilon_{t}$ is conditionally heteroskedastic. In order to derive conditional volatility specifications, it is necessary to specify the stochastic processes underlying the returns shocks, $\varepsilon_{t}$. 
Consider the following random coefficient autoregressive process of order one:

$$
\varepsilon_{t}=\phi_{t} \varepsilon_{t-1}+\eta_{t}
$$

where

$$
\begin{gathered}
\phi_{t} \sim \operatorname{iid}(0, \alpha), \\
\eta_{t} \sim \operatorname{iid}(0, \omega) .
\end{gathered}
$$

Tsay (1987) [6] showed that the ARCH(1) model of Engle (1982) [1] could be derived from Equation (2) as:

$$
h_{t}=E\left(\varepsilon_{t}^{2} \mid I_{t-1}\right)=\omega+\alpha \varepsilon_{t-1}^{2}
$$

where $h_{t}$ is conditional volatility, and $I_{t-1}$ is the information set at time $t-1$. The use of an infinite lag length for the random coefficient autoregressive process in Equation (2), with appropriate restrictions on the random coefficients, can be shown to lead to the GARCH model of Bollerslev (1986) [2].

As the ARCH and GARCH models are symmetric, in that positive and negative shocks of equal magnitude have identical effects on conditional volatility, there is no asymmetry, and hence also no leverage, whereby negative shocks increase conditional volatility and positive shocks decrease conditional volatility (see Black (1976) [8]).

It is worth noting that at least one of $\omega$ or $\alpha$ must be positive for conditional volatility to be positive, with $\omega>0$ and $\alpha>0$ regarded as sufficient conditions for positivity of conditional volatility. From the specification of Equation (2), it is clear that both $\omega$ and $\alpha$ should be positive as they are the variances of two different stochastic processes.

From a practical perspective, a failure to impose the positivity restrictions on the parameters can increase the probability of obtaining negative estimates of conditional volatility. For example, in the current version R2014a of MATLAB, no positivity is imposed in estimating the parameters of GARCH. Similar comments apply to other standard econometric, financial econometric and statistical software packages, and to the GJR and EGARCH models that are discussed below.

\subsection{Random Coefficient Autoregressive Process and GJR}

The GJR model of Glosten, Jagannathan and Runkle (1992) [3] can be derived as a simple extension of the random coefficient autoregressive process in Equation (2), with an indicator variable $I\left(\varepsilon_{t-1}\right)$ that distinguishes between the different effects of positive and negative returns shocks on conditional volatility, namely:

$$
\varepsilon_{t}=\phi_{t} \varepsilon_{t-1}+\psi_{t} I\left(\varepsilon_{t-1}\right)+\eta_{t}
$$

where

$$
\begin{gathered}
\phi_{t} \sim \operatorname{iid}(0, \alpha), \\
\psi_{t} \sim \operatorname{iid}(0, \gamma), \\
\eta_{t} \sim \operatorname{iid}(0, \omega), \\
I\left(\varepsilon_{t-1}\right)=1 \text { when } \varepsilon_{t-1}<0, \\
I\left(\varepsilon_{t-1}\right)=0 \text { when } \varepsilon_{t-1} \geq 0 .
\end{gathered}
$$


The conditional expectation of the squared returns shocks in (3), which is typically referred to as the GJR (or threshold GARCH) model, can be shown to be an extension of Equation (3), as follows:

$$
h_{t}=E\left(\varepsilon_{t}^{2} \mid I_{t-1}\right)=\omega+\alpha \varepsilon_{t-1}^{2}+\gamma I\left(\varepsilon_{t-1}\right) \varepsilon_{t-1}^{2}
$$

The use of an infinite lag length for the random coefficient autoregressive process in Equation (4), with appropriate restrictions on the random coefficients, can be shown to lead to the standard GJR model with lagged conditional volatility.

As GARCH is nested within GJR, the interpretation of the coefficients in the two models is essentially the same, apart from the parameter associated with asymmetry.

It is worth noting that at least one of $(\omega, \alpha, \gamma)$ must be positive for conditional volatility to be positive, with $\omega>0, \alpha>0$ and $\gamma>0$ regarded as sufficient conditions for positivity of conditional volatility. From the specification of Equation (4), it is clear that all three parameters should be positive as they are the variances of three different stochastic processes.

The GJR model is asymmetric, in that positive and negative shocks of equal magnitude have different effects on conditional volatility. Therefore, asymmetry exists for GJR if:

asymmetry for GJR: $\gamma>0$.

A special case of asymmetry is leverage, which is the negative correlation between returns shocks and subsequent shocks to volatility (see Black (1976) [8]). The conditions for leverage in the GJR model in Equation (5) are:

leverage for GJR: $\alpha<0$ and $\alpha+\gamma>0$.

It is clear that leverage is not possible for GJR as both $\alpha$ and $\gamma$, which are the variances of two stochastic processes, must be positive.

As in the case of GARCH, the positivity restrictions on the parameters of GJR are typically not imposed in estimation using standard econometric, financial econometric and statistical software packages.

\subsection{Random Coefficient Complex Nonlinear Moving Average Process and EGARCH}

Another conditional volatility model that can accommodate asymmetry is the EGARCH model of Nelson (1990, 1991) [4,5]. McAleer and Hafner (2014) [7] showed that EGARCH could be derived from a random coefficient complex nonlinear moving average (RCCNMA) process, as follows:

$$
\varepsilon_{t}=\phi_{t} \sqrt{\left|\eta_{t-1}\right|}+\psi_{t} \sqrt{\eta_{t-1}}+\eta_{t}
$$

where

$$
\begin{aligned}
& \phi_{t} \sim \operatorname{iid}(0, \alpha), \\
& \psi_{t} \sim \operatorname{iid}(0, \gamma), \\
& \eta_{t} \sim \operatorname{iid}(0, \omega),
\end{aligned}
$$

$\sqrt{\eta_{t-1}}$ is a complex-valued function of $\eta_{t-1}$. 
The conditional variance of the squared returns shocks in Equation (6) is given as:

$$
h_{t}=E\left(\varepsilon_{t}^{2} \mid I_{t-1}\right)=\omega+\alpha\left|\eta_{t-1}\right|+\gamma \eta_{t-1}
$$

It is worth noting that the transformation of $h_{t}$ in Equation (7) is not logarithmic, but the approximation given by:

$$
\log h_{t}=\log \left(1+\left(h_{t}-1\right)\right) \approx h_{t}-1
$$

can be used to replace $h_{t}$ in Equation (7) with $1+\log h_{t}$. The use of an infinite lag for the RCCNMA process in Equation (6) would yield, after suitable logarithmic approximation, the standard EGARCH model with lagged conditional volatility.

EGARCH differs from GARCH and GJR in that, given the logarithmic transformation, no sign restrictions on $(\omega, \alpha, \gamma)$ are necessary for conditional volatility to be positive. However, it is clear from the RCCNMA process in Equation (6) that all three parameters should be positive as they are the variances of three different stochastic processes. Therefore, asymmetry exists for EGARCH if:

$$
\text { asymmetry for EGARCH: } \gamma>0 \text {. }
$$

The conditions for leverage in the EGARCH model in Equation (7) are:

$$
\text { leverage for EGARCH: } \gamma<0 \text { and } \gamma<\alpha<-\gamma \text {. }
$$

As acknowledged in McAleer and Hafner (2014) [7], leverage is not possible as both $\alpha$ and $\gamma$, which are the variances of two stochastic processes, must be positive.

As EGARCH is non-nested with both GARCH and GJR, the interpretation of the coefficients in EGARCH is not the same as in the other two conditional volatility models, although the definitions of asymmetry and leverage are identical. The derivations in Section 2 are intended to keep the number of iid processes to a minimum for ease of presentation.

As in the case of GARCH and GJR, the positivity restrictions on the parameters of GJR are typically not imposed in estimation using standard econometric, financial econometric and statistical software packages.

\section{Conclusions}

The paper was concerned with the three most widely-used univariate conditional volatility models, namely the GARCH, GJR (or threshold GARCH) and EGARCH models. These models are important in estimating and forecasting volatility, as well as in capturing asymmetry, which is the different effects on conditional volatility of positive and negative effects of equal magnitude, and purportedly in capturing leverage, which is the negative correlation between returns shocks and subsequent shocks to volatility.

As discussed in Section 2, a failure to impose the positivity restrictions on the parameters of the conditional volatility models can increase the probability of obtaining negative estimates of conditional volatility. In standard econometric, financial econometric and statistical software packages, it is typically the case that no positivity is imposed in estimating the parameters of the three most popular conditional volatility models. 
As there seems to be some confusion in the literature between asymmetry and leverage, as well as which asymmetric models are purported to be able to capture leverage, the purpose of the paper was three-fold, namely, (1) to derive the GJR model from a random coefficient autoregressive process, with appropriate regularity conditions; (2) to show that leverage is not possible in the GJR and EGARCH models; and (3) to present the interpretation of the parameters of the three popular univariate conditional volatility models in a unified manner.

\section{Acknowledgements}

The author wishes to thank Massimiliano Caporin, Marc Paolella, Pawel Polak, the Editor-in-Chief, Kerry Patterson, and a reviewer for helpful comments and suggestions. For financial support, the author wishes to acknowledge the Australian Research Council and the National Science Council, Taiwan.

\section{Conflicts of Interest}

The author has no conflicts of interest.

\section{References}

1. Engle, R.F. Autoregressive conditional heteroscedasticity with estimates of the variance of United Kingdom inflation. Econometrica 1982, 50, 987-1007.

2. Bollerslev, T. Generalised autoregressive conditional heteroscedasticity. J. Econom. 1986, 31, 307-327.

3. Glosten, L.R.; Jagannathan, R.; Runkle, D.E. On the relation between the expected value and volatility of nominal excess return on stocks. J. Financ.1992, 46, 1779-1801.

4. Nelson, D.B. ARCH models as diffusion approximations. J. Econom. 1990, 45, 7-38.

5. Nelson, D.B. Conditional heteroskedasticity in asset returns: A new approach. Econometrica 1991, 59, 347-370.

6. Tsay, R.S. Conditional heteroscedastic time series models. J. Am. Stat. Assoc. 1987, 82, 590-604.

7. McAleer, M.; Hafner, C. A one line derivation of EGARCH. Econometrics 2014, 2, 92-97.

8. Black, F. Studies of Stock Market Volatility Changes. In Proceedings of the American Statistical Association, Business and Economic Statistics Section, Washington, DC, USA, 1976; pp. 177-181.

(C) 2014 by the authors; licensee MDPI, Basel, Switzerland. This article is an open access article distributed under the terms and conditions of the Creative Commons Attribution license (http://creativecommons.org/licenses/by/4.0/). 